\title{
$K(n)$ Chern approximations of some finite groups
}

\author{
BJÖRN SCHUSTER
}

\begin{abstract}
A few examples of 2-groups are presented whose Morava $\mathrm{K}$-theory is determined by representation theory. By contrast, a 3-primary example shows that in general relations arising from representation theory do not suffice to calculate the Chern subring of $K(n)^{*}(B G)$.
\end{abstract}

55N20, 55R35; 55T25

\section{Introduction}

Let $E$ denote a complex oriented cohomology theory and $G$ a finite group. As any complex oriented theory comes with a theory of Chern classes of complex vector bundles, complex representations offer a convenient source of $E$-cohomology classes of $B G$, the classifying space of the group $G$. In many examples, one knows that Chern classes suffice to generate $E^{*}(B G)$, and it is natural to ask to what extend the relations among them follow from representation theory, too.

This question is closely related to the problem of determining the so-called Chern approximation of $E^{*}(B G)$, a concept introduced by Strickland [9]: take all nontrivial irreducible complex representations $\rho$ of $G$, assign indeterminates to the Chern classes of such $\rho$, and divide out by the relations obtained from the product structure of the representation ring and all $\lambda$-operations (for a precise definition see below). Strickland then studies the resulting object in geometric terms, ie, the associated formal scheme over the formal group $E^{0} \mathbb{C} P^{\infty}$.

We shall work with $E=K(n)$, the $n$-th $\bmod p$ Morava K-theory with coefficients $K(n)^{*}=\mathbb{F}_{p}\left[v_{n}, v_{n}^{-1}\right]$, where $v_{n}$ has degree $-2\left(p^{n}-1\right)$. Our calculations show that for some 2-groups $G$, the $K(n)$ Chern approximation coincides with $K(n)^{*}(B G)$. To prove such results one has to perform two steps: First, establish that $K(n)^{*}(B G)$ is generated by Chern classes of complex representations; in the cases we shall study this is already in the literature. Secondly, one has to show that the relations implied by the structure of the representation ring $R G$ suffice. To that end it is enough to produce an upper bound for the rank of the resulting module (which spares us the necessity to use 
Gröbner basis methods), and compare it to the rank of $K(n)^{*}(B G)$, which by the first step is given by the Euler characteristic formula of Hopkins, Kuhn and Ravenel [4].

Although to some extent motivated by the problem of finding the ring structure of $K(n)^{*}(B G)$, this is not the primary purpose of the present paper. For most of the groups considered here, the multiplicative structure of $K(n)^{*}(B G)$ has already been determined using more efficient transfer methods; see Bakuradze [1] and Bakuradze and Vershinin [3]. What interests us here is the question whether $K(n)^{*}(B G)$ is already determined, as $K(n)^{*}$-algebra, by the representation theory of $G$. For our 2-group examples this is true; however, for the nonabelian group of order 27 and exponent 3 , the answer is negative. This latter result has been known to Strickland for some time.

The paper is organised as follows. We start with a brief review of Chern approximations for $K(n)$-theory. The account given here is a "poor man's version" of the original, inasmuch we forego all mention of the finer geometric structure. Next we record a few useful formulas, and the later sections contain the calculations for the individual groups: dihedral, quaternion, semidihedral and quasidihedral groups, and one 3-primary example.

Acknowledgements We are indebted to the first referee for useful hints regarding the proof of Lemma 3.1(ii), and to both referees for suggesting many improvements to the exposition.

\section{Chern approximations for $K(n)$}

Let $G$ be a finite group. Suppose $\mu$ and $\rho$ are complex representations of dimension $m$ and $r$, respectively. Let $\sigma_{i}(s)$ and $\sigma_{j}(t)$ denote the elementary symmetric functions in $s_{1}, \ldots, s_{m}$ and $t_{1}, \ldots, t_{r}$. Recall that the $k$-th Chern class of $\rho$, say, can be identified with the coefficient of $X^{n-k}$ in $\prod_{i=1}^{r}\left(X-t_{i}\right)$. Furthermore, the coefficient of $X^{k}$ in

$$
\prod_{\substack{1 \leq i \leq m \\ 1 \leq j \leq r}}\left(1+X\left(s_{i}+{ }_{F} t_{j}\right)\right)
$$

is a polynomial in the $\sigma_{i}(s)$ and $\sigma_{j}(t)$, say $P_{k}\left(\sigma_{1}(s), \ldots, \sigma_{m}(s) ; \sigma_{1}(t), \ldots, \sigma_{r}(t)\right)$. Here and below we write $x+F y$ to denote the formal sum of $x$ and $y$.

Similarly, the coefficient of $X^{k}$ in

$$
\prod_{i_{1}<\cdots<i_{q}}\left(1+X\left(s_{i_{1}}+{ }_{F} s_{i_{2}}+{ }_{F} \cdots+{ }_{F} s_{i_{q}}\right)\right)
$$

is a polynomial $L_{k}$ in the $\sigma_{i}(s)$. 
The splitting principle implies that these power series determine then Chern classes of products and exterior powers:

Proposition $2.1 \quad(1) \quad c_{k}(\mu \otimes \rho)=P_{k}\left(c_{1}(\mu), \ldots, c_{m}(\mu) ; c_{1}(\rho), \ldots, c_{r}(\rho)\right)$.

(2) $c_{k}\left(\lambda^{q} \mu\right)=L_{k}\left(c_{1}(\mu), \ldots, c_{m}(\mu)\right)$.

Next, recall Adams operations on the representation ring. Let $\mu$ be a representation of dimension $m$; set $\lambda_{t}(\mu)=\sum_{i \geq 0} \lambda^{i}(\mu) t^{i}$ (where $\lambda^{0} \mu=1$ ), and define

$$
\psi_{t}(\mu)=m-\frac{t}{\lambda_{-t}(\mu)} \frac{d}{d t} \lambda_{-t}(\mu) .
$$

Then $\psi^{l} \mu$ is the coefficient of $t^{l}$ in $\psi_{t}(\mu)$. There are the well-known formulae linking Adams operations and exterior powers via the Newton polynomials; in particular, $\psi^{k}(\mu)=\mu^{k}$ for any line bundle (one-dimensional representation). Hence for a direct sum of line bundles one has

$$
c_{k}\left(\psi^{l}\left(\mu_{1} \oplus \cdots \oplus \mu_{m}\right)\right)=c_{k}\left(\mu_{1}^{l} \oplus \cdots \oplus \mu_{m}^{l}\right)=\sigma_{k}\left([l]\left(x_{1}\right), \ldots,[l]\left(x_{m}\right)\right)
$$

where $x_{i}=c_{1}\left(\mu_{i}\right)$. Thus:

Proposition 2.2 For the $K(n)$ Chern classes one has $c_{k}\left(\psi^{p^{r}} \mu\right)=c_{k}(\mu)^{p^{r n}}$.

Definition 2.3 (Strickland [9]) Let $G$ be a finite group. Let $\rho_{1}, \ldots, \rho_{k}$ be the distinct nontrivial irreducible complex representations of $G$. For each $\rho_{i}$, choose indeterminates $c_{l, i}, 1 \leq l \leq \operatorname{dim}\left(\rho_{i}\right)$. Define $C(G ; K(n))$ to be the quotient of the $K(n)^{*}$-algebra on the $c_{l, i}$ by the relations imposed by Proposition 2.1.

As a consequence of Proposition 2.2, one gets the following special case of [9, Corollary 10.3]. Our proof is but a paraphrase of the argument given there.

Corollary 2.4 For any finite group $G$, the rank of $C(G ; K(n))$ over $K(n)^{*}$ is finite.

Proof It suffices to show that all generators of $C(G ; K(n))$ are nilpotent. Let $e$ be the exponent of the group $G$ and $p^{r}$ its $p$-part, ie, $e=p^{r} f$ with $f$ coprime to $p$. Then $\psi^{e}(\mu)=\operatorname{dim}(\mu)$ for any representation $\mu$ of $G$. Thus for $k \geq 1$, one has $0=c_{k}\left(\psi^{e} \mu\right)=c_{k}\left(\psi^{p^{r}} \psi^{f} \mu\right)=c_{k}\left(\psi^{f} \mu\right)^{p^{r n}}$. Now let $c_{\bullet}$ denote the total Chern class; since we are working modulo $p$, we find that

$$
1=c_{\bullet}\left(\psi^{f} \mu\right)^{p^{r n}}=c_{\bullet}\left(p^{r n} \psi^{f} \mu\right)=c_{\bullet}\left(\psi^{f}\left(p^{r n} \mu\right)\right)
$$

(using additivity) and thus $c_{k}\left(\psi^{f}\left(p^{r n} \mu\right)\right)=0$ for all $k \geq 1$. But when $f$ is coprime to $p$, the series $[f](x)$ is an automorphism of the formal group law; thus $c_{k} \psi^{f}=0$ for all $k>0$ if and only if $c_{k}=0$ for all $k>0$. This implies $1=c_{\bullet}\left(p^{r n} \mu\right)=c_{\bullet}(\mu)^{p^{r n}}$, whence the claim. 
There is an obvious map

$$
\operatorname{ch}_{G}: C(G ; K(n)) \longrightarrow K(n)^{*}(B G)
$$

assigning to $c_{k, i}$ the Chern class $c_{k}\left(\rho_{i}\right)$. In general, this map is neither injective not surjective: an example of noninjectivity is given in Section 7, whereas it certainly fails to be onto whenever $K(n)^{*}(B G)$ is not generated by Chern classes, as happens for $G=A_{4}$ at $p=2$ (there are $p$-group examples, too).

Definition 2.5 We call the Chern approximation of $G$ exact if $\operatorname{ch}_{G}$ is an isomorphism.

\section{Some formulas}

From now on we shall work with $K(n)^{*}(-) \otimes_{K(n)^{*}} \mathbb{F}_{p}$, ie, we set $v_{n}=1$.

We start by giving two approximations to the formal group law of Morava K-theory.

Lemma 3.1 (i) For any $p$,

$$
x_{1}+F x_{2}=x_{1}+x_{2}-\frac{1}{p} \sum_{i=1}^{p-1}\left(\begin{array}{c}
p \\
i
\end{array}\right) x_{1}^{p^{n-1} i} x_{2}^{p^{n-1}(p-i)} \bmod \left(\left(x_{1} x_{2}\right)^{p^{2 n-2}}\right) .
$$

If $p$ is odd, this equality holds modulo $\left(\left(x_{1}+x_{2}\right) x_{1} x_{2}\right)^{p^{2 n-2}}$.

(ii) Let $p=2$. Then

$x_{1}+F_{2} x_{2}$

$$
=x_{1}+x_{2}+\left(x_{1} x_{2}+\left(x_{1}+x_{2}\right)\left(x_{1} x_{2}\right)^{2^{n-1}}\right)^{2^{n-1}} \bmod \left(\left(\left(x_{1}+x_{2}\right) x_{1} x_{2}\right)^{2^{2 n-2}}\right) .
$$

Proof Part (i) is stated in Bakuradze and Priddy [2, Lemma 5.3] and (ii) is claimed in Bakuradze and Vershinin [3, Lemma 2.2 (ii)], but, as the referee pointed out, the explanation provided there falls short of a full proof. We therefore give an argument which surely must be the one the authors of [3] had in mind. Since we need the notation anyway, we also show (i), the proof being essentially the one from [2].

Specialising Theorem 4.3.9 of Ravenel's green book [6] to the case where $v_{n}=1$ and $v_{i}=0$ for $n \neq i$ and simplifying it using [6, Lemma 4.3.8. (b)] gives

$$
\sum^{F} x_{i}=\sum_{k \geq 0}^{F} w_{k}\left(x_{1}, x_{2}, \ldots\right)^{p^{(n-1) k}}
$$


for any number of variables, where the Witt polynomials $w_{k}\left(x_{1}, x_{2}, \ldots\right) \in \mathbb{Z}\left[x_{1}, x_{2}, \ldots\right]$ are characterised by $w_{0}=\sigma_{1}\left(x_{1}, x_{2}, \ldots\right)$ and

$$
\sum_{i} x_{i}^{p^{k}}=\sum_{j=0}^{k} p^{j} w_{j}^{p^{k-j}}
$$

By construction, the Witt polynomials are symmetric, and in the case of two variables $x_{1}, x_{2}$ one has

$$
w_{1}=-\frac{1}{p} \sum_{j=1}^{p-1}\left(\begin{array}{c}
p \\
j
\end{array}\right) x_{1}^{j} x_{2}^{p-j}
$$

In particular, $w_{1}$ is divisible by $x_{1} x_{2}$, and by induction, all $w_{k}\left(x_{1}, x_{2}\right)$ are in $\left(x_{1} x_{2}\right)$. More precisely, for $p$ odd one even has $w_{1} \in\left(x_{1} x_{2}\left(x_{1}+x_{2}\right)\right)$, whence the same holds for all $w_{k}$. For $p=2$, however, $w_{1}\left(x_{1}, x_{2}\right)=x_{1} x_{2}$, but still $w_{k} \in\left(x_{1} x_{2}\left(x_{1}+x_{2}\right)\right)$ for $k \geq 2$. To see this, denote by $q_{m}$ the power sum $x_{1}^{m}+x_{2}^{m}$. Newton's identities reduce to $q_{m+1}=\left(x_{1}+x_{2}\right) q_{m}-x_{1} x_{2} q_{m-1}$. This gives $q_{2^{k}}=\left(x_{1}+x_{2}\right)^{2^{k}}+2\left(x_{1} x_{2}\right)^{2^{k-1}}$ $\bmod \left(x_{1} x_{2}\left(x_{1}+x_{2}\right)\right)$ by induction, hence

$$
\begin{aligned}
2^{k} w_{k}\left(x_{1}, x_{2}\right) & =q_{2^{k}}-\sum_{i=0}^{k-1} 2^{i} w_{i}^{2^{k-i}} \\
& =\left(x_{1}+x_{2}\right)^{2^{k}}+2\left(x_{1} x_{2}\right)^{2^{k-1}}-w_{0}^{2^{k}}-2 w_{1}^{2^{k-1}} \bmod \left(x_{1} x_{2}\left(x_{1}+x_{2}\right)\right) \\
& =0 \bmod \left(x_{1} x_{2}\left(x_{1}+x_{2}\right)\right) .
\end{aligned}
$$

Thus

$$
x_{1}+F x_{2}=\left(x_{1}+x_{2}\right)+F\left(-\frac{1}{p} \sum_{i=1}^{p-1}\left(\begin{array}{c}
p \\
i
\end{array}\right) x_{1}^{p-i} x_{2}^{i}\right)^{p^{n-1}} \bmod \left(\left(x_{1} x_{2}\right)^{p^{2 n-2}}\right) .
$$

Writing now $y_{1}=w_{0}\left(x_{1}, x_{2}\right)$ and $y_{2}=w_{1}\left(x_{1}, x_{2}\right)^{p^{n-1}}$, repeating the same argument leads to

$$
x_{1}+F_{2} x_{2}=w_{0}\left(y_{1}, y_{2}\right)+F w_{1}\left(y_{1}, y_{2}\right)^{p^{n-1}} \bmod \left(\left(y_{1} y_{2}\right)^{p^{2 n-2}}\right),
$$

so certainly modulo $\left(x_{1} x_{2}\right)^{p^{2 n-2}}$. But modulo $p$, one has

$$
w_{1}\left(y_{1}, y_{2}\right)^{p^{n-1}}=\left(-\frac{1}{p} \sum_{i=1}^{p-1}\left(\begin{array}{c}
p \\
i
\end{array}\right)\left(x_{1}+x_{2}\right)^{p-i} w_{1}\left(x_{1}, x_{2}\right)^{p^{n-1} i}\right)^{p^{n-1}}
$$

and this is zero modulo $\left(x_{1} x_{2}\right)^{p^{2 n-2}}$; the claim follows. For $p$ odd, the second claim of (i) follows from the same calculation, since $w_{1}\left(x_{1}, x_{2}\right)$ is in $\left(x_{1} x_{2}\left(x_{1}+x_{2}\right)\right)$. 
Let now $p=2$. Then, as $w_{2}\left(x_{1}, x_{2}\right)=x_{1} x_{2}$, we can no longer argue as before. On the other hand, by part (i),

$$
\begin{aligned}
w_{0}\left(y_{1}, y_{2}\right)+{ }_{F} w_{1}\left(y_{1}, y_{2}\right)^{2^{n-1}}=w_{0}\left(y_{1}, y_{2}\right)+ & w_{1}\left(y_{1}, y_{2}\right)^{2^{n-1}} \\
& +w_{0}\left(y_{1}, y_{2}\right)^{2^{n-1}} w_{1}\left(y_{1}, y_{2}\right)^{2^{2 n-2}}
\end{aligned}
$$

$\bmod \left(w_{0}\left(y_{1}, y_{2}\right)^{2^{2 n-2}} w_{1}\left(y_{1}, y_{2}\right)^{2^{3 n-3}}\right)$, in particular mod $\left.\left(x_{1} x_{2}\left(x_{1}+x_{2}\right)\right)^{2^{2 n-2}}\right)$. But the third summand is also in $\left(\left(x_{1} x_{2}\left(x_{1}+x_{2}\right)\right)^{2^{2 n-2}}\right)$, so we finally arrive at

$$
\begin{aligned}
& x_{1}+{ }_{F} x_{2} \\
& =w_{0}\left(y_{1}, y_{2}\right)+w_{1}\left(y_{1}, y_{2}\right)^{2^{n-1}} \bmod \left(x_{1} x_{2}\left(x_{1}+x_{2}\right)\right)^{2^{2 n-2}} \\
& =x_{1}+x_{2}+\left(x_{1} x_{2}\right)^{2^{n-1}}\left(\left(x_{1}+x_{2}\right)\left(x_{1} x_{2}\right)^{2^{n-1}}\right)^{2^{n-1}} \bmod \left(x_{1} x_{2}\left(x_{1}+x_{2}\right)^{2^{2 n-2}}\right)
\end{aligned}
$$

as claimed.

Lemma 3.2 Let $p=2$ and $\varepsilon, \tau \in R G$ be of dimension 1 and 2, respectively. Assume further that $\varepsilon^{2}=1$. Set $y=c_{1}(\varepsilon)$ and $c_{i}=c_{i}(\tau)(i=1,2)$. Then

(i) $y^{2^{n}}=0$;

(ii) $c_{1}(\varepsilon \tau)=c_{1}+\left(y c_{1}\right)^{2^{n-1}}$;

(iii) $c_{2}(\varepsilon \tau)=y^{2}+y c_{1}+c_{2}+y^{2^{n-1}+1} c_{1}^{2^{n-1}}+y^{2^{n-1}} \sum_{k=1}^{n-1} c_{1}^{2^{n-1}-2^{k}+1} c_{2}^{2^{k-1}}$.

If in addition $\varepsilon \tau=\tau$, then

(iv) $\left(y c_{1}\right)^{2^{n-1}}=0$;

(v) $y c_{1}^{2}=y^{3}$;

(vi) $y c_{1}=y^{2}+\sum_{k=1}^{n-1} y^{2^{n}-2^{k}+1} c_{2}^{2^{k-1}}$.

Proof Part (i) is immediate from [2] $(y)=y^{2^{n}}$. For (ii) write $\tau=\xi_{1}+\xi_{2}$ as a sum of line bundles and $x_{i}=c_{1}\left(\xi_{i}\right)$. By the splitting principle, we may calculate in $\mathbb{F}_{2}[y] /\left(y^{2^{n}}\right) \otimes \mathbb{F}_{2}\left[x_{1}, x_{2}\right]^{\Sigma_{2}}$, identifying $c_{1}$ with $x_{2}+x_{2}$ and $c_{2}$ with $x_{1} x_{2}$. Then by (i),

$c_{1}(\varepsilon \tau)=\left(y+{ }_{F} x_{1}\right)+\left(y+{ }_{F} x_{2}\right)=\left(x_{1}+x_{2}\right)+y^{2^{n-1}}\left(x_{1}+x_{2}\right)^{2^{n-1}}=c_{1}+\left(y c_{1}\right)^{2^{n-1}}$,

using Lemma 3.1(i). Similarly,

$c_{2}(\varepsilon \tau)$

$=\left(y+{ }_{F} x_{2}\right)\left(y+{ }_{F} x_{2}\right)$

$=y^{2}+y\left(x_{1}+x_{2}\right)+x_{1} x_{2}+y^{2^{n-1}+1}\left(x_{1}+x_{2}\right)^{2^{n-1}}+y^{2^{n-1}}\left(x_{1}^{2^{n-1}} x_{2}+x_{1} x_{2}^{2^{n-1}}\right)$ 
which gives (iii). Part (iv) is clear. Furthermore, if $\varepsilon \tau=\tau$, (iii) reads as

$$
c_{1}=y+y^{2^{n-1}-1} \sum_{k=1}^{n-1} c_{1}^{2^{n-1}-2^{k}+1} c_{2}^{2^{k-1}} \bmod \operatorname{ann}(y)
$$

whence

$$
c_{1}^{2}=y^{2}+y^{2^{n}-2} c_{1}^{2} \sum_{k=1}^{n-1} c_{1}^{2^{n}-2^{k+1}} c_{2}^{2^{k}}=y^{2} \bmod \operatorname{ann}(y)
$$

which implies (v). Finally, (vi) is a consequence of (iii) and (v).

We also record the following formulas, which can be verified in similar style using Lemma 3.1(ii).

Lemma 3.3 Let $p=2$ and $\tau \in R G$ of dimension 2. Set $c_{i}=c_{i}(\tau)$. Then

(i) $c_{1}\left(\tau^{2}\right)=c_{1}^{2^{n}}$;

(ii) $c_{2}\left(\tau^{2}\right)=c_{1}^{2}+c_{1}^{2^{n}} c_{2}^{2^{2 n-1}} \bmod \left(c_{1} c_{2}\right)^{2^{2 n-1}}$;

(iii) $c_{3}\left(\tau^{2}\right)=c_{1}^{2^{n}+2}+c_{1}^{2^{n}} c_{2}^{2^{n}}+c_{1}^{2^{n+1}} c_{2}^{2^{2 n-1}} \bmod c_{1}^{2^{2 n-1}+2^{n}} c_{2}^{2^{2 n-1}}$ $=c_{1}^{2^{n}} c_{2}^{2^{n}} \bmod c_{1}^{2^{n}+1}$;

(iv) $c_{4}\left(\tau^{2}\right)=c_{1}^{2} c_{2}^{2^{n}}+c_{2}^{2^{n+1}}+c_{1}^{2^{n}} c_{2}^{2^{2 n-1}+2^{n}} \bmod c_{1}^{2^{2 n-1}}$;

(v) $c_{1}\left(\lambda^{2} \tau\right)=c_{1}+c_{2}^{2^{n-1}}+c_{1}^{2^{n-1}} c_{2}^{2^{2 n-2}} \bmod \left(c_{1} c_{2}\right)^{2^{2 n-2}}$.

\section{The groups $D_{8}$ and $Q_{8}$}

We start with dihedral and quaternion groups of order 8 . Not only is this the simplest case, it shall also furnish us with certain identities useful later on. The quaternion case was already treated in detail in Strickland [9, Section 15] and is only included here since it does not mean any extra effort.

Both groups have isomorphic complex representation rings, but they differ in the $\lambda$-structure. To fix notation, we use the following presentations of $D_{8}$ and $Q_{8}$ : both are two generator groups, on $g_{1}, g_{2}$, say, with relators

$$
\begin{aligned}
g_{1}^{2}, g_{2}^{2},\left[g_{1}, g_{2}\right]^{2} & \text { for } D_{8}, \\
g_{1}^{4}, g_{1}^{2} g_{2}^{2}, g_{1} g_{2}^{-1} g_{1} g_{2} & \text { for } Q_{8} .
\end{aligned}
$$


There are four one-dimensional representations and one irreducible of dimension two. Let $\gamma_{j}$ be defined by $\gamma_{j}\left(g_{k}\right)=(-1)^{\delta_{j k}}(j, k=1,2)$, and $\Delta=\operatorname{Ind}_{\left\langle g_{1} g_{2}\right\rangle}^{G}(\beta)$ where $\beta\left(g_{1} g_{2}\right)=i$. Then one has $\gamma_{j}^{2}=1, \gamma_{j} \Delta=\Delta, \Delta^{2}=1+\gamma_{1}+\gamma_{2}+\gamma_{1} \gamma_{2}$, and

$$
\lambda^{2} \Delta= \begin{cases}\gamma_{1} \gamma_{2} & \text { for } D_{8}, \\ 1 & \text { for } Q_{8} .\end{cases}
$$

Let $y_{i}=c_{1}\left(\gamma_{i}\right)(i=1,2)$ and $c_{j}=c_{j}(\Delta)(j=1,2)$. Then it is known that $K(n)^{*}(B G)$ is multiplicatively generated by $y_{1}, y_{2}$ and $c_{2}$; see Tezuka and Yagita [10] and Schuster and Yagita [8]. The first relations are easy: from $\gamma_{i}^{2}=1$ we immediately obtain

$$
y_{1}^{2^{n}}=0, \quad y_{2}^{2^{n}}=0 .
$$

Now to $\gamma_{i} \Delta=\Delta$ : by Lemma 3.2, one obtains

$$
\begin{aligned}
& \left(y_{i} c_{1}\right)^{2^{n-1}}=0 \\
& y_{i} c_{1}=y_{i}^{2}+\sum_{k=1}^{n-1} y_{i}^{2^{n}-2^{k}+1} c_{2}^{2^{k-1}} .
\end{aligned}
$$

We intend to use $\Delta^{2}=1+\gamma_{1}+\gamma_{2}+\gamma_{1} \gamma_{2}$ next: one has $c_{1}\left(\Delta^{2}\right)=c_{1}(\Delta)^{2^{n}}$ by Lemma 3.3(i), hence

$$
c_{1}^{2^{n}}=y_{1}+y_{2}+\left(y_{1}+F y_{2}\right)=\left(y_{1} y_{2}\right)^{2^{n-1}} .
$$

Now (3) can be restated as

$$
c_{1}+y_{i}+\sum_{k=1}^{n-1} y_{i}^{2^{n}-2^{k}} c_{2}^{2^{k-1}} \in \operatorname{ann}\left(y_{i}\right)
$$

Using (1), raising this to the power $2^{n}$ yields $c_{1}^{2^{n}} \in \operatorname{ann}\left(y_{i}\right)$, which in turn implies

$$
y_{i}\left(y_{1} y_{2}\right)^{2^{n}}=y_{i} c_{1}^{2^{n}}=0
$$

and thus, using (3) again,

$$
\begin{gathered}
c_{1}^{2^{n}+1}=\left(y_{1} y_{2}\right)^{2^{n-1}} c_{1}=y_{1}^{2^{n-1}-1} y_{2}^{2^{n-1}}\left(y_{1}^{2}+\sum_{k=1}^{n-1} y_{1}^{2^{n}-2^{k}+1} c_{2}^{2^{k-1}}\right) \\
=y_{1}^{2^{n-1}+1} y_{2}^{2^{n-1}}=y_{1} c_{1}^{2^{n}}=0 .
\end{gathered}
$$

By Lemma 3.2(v),

$$
y_{i} c_{1}^{2}=y_{i}^{3}
$$


which implies $y_{1}^{3} y_{2}=y_{1} y_{2} c_{1}^{2}=y_{1} y_{2}^{3}$. This gives

$$
\begin{aligned}
y_{1} y_{2} c_{1}=y_{1}^{2} y_{2}+\sum_{k=1}^{n-1} y_{1}^{2^{n}-2^{k}+1} y_{2} c_{2}^{2^{k-1}} & =y_{1}^{2} y_{2}+\sum_{k=1}^{n-1} y_{1} y_{2}^{2^{n}-2^{k}-1} c_{2}^{2^{k-1}} \\
& =y_{1}^{2} y_{2}+y_{1} y_{2} c_{1}+y_{1} y_{2}^{2},
\end{aligned}
$$

hence

$$
y_{1}^{2} y_{2}=y_{1} y_{2}^{2} .
$$

Furthermore, since we may calculate modulo $c_{1}^{2^{n}+1}$ by (6), Lemma 3.3 implies

$$
c_{2}\left(\Delta^{2}\right)=c_{1}^{2}+c_{1}^{2^{n}} c_{2}^{2^{2 n-1}} .
$$

On the other hand

$c_{2}\left(1+\gamma_{1}+\gamma_{2}+\gamma_{1} \gamma_{2}\right)=y_{1} y_{2}+\left(y_{1}+y_{2}\right)\left(y_{1}+y_{2}+y_{1}^{2^{n-1}} y_{2}^{2^{n-1}}\right)=y_{1}^{2}+y_{1} y_{2}+y_{2}^{2}$ using (8), thus

$$
c_{1}^{2}=y_{1}^{2}+y_{1} y_{2}+y_{2}^{2}+\left(y_{1} y_{2}\right)^{2^{n-1}} c_{2}^{2^{2 n-1}} .
$$

Also, modulo $c_{1}^{2^{n}+1}$ one has $c_{3}\left(\Delta^{2}\right)=c_{1}^{2^{n}} c_{2}^{2^{n}}$ and $c_{3}\left(1+\gamma_{1}+\gamma_{2}+\gamma_{1} \gamma_{2}\right)=y_{1} y_{2}\left(y_{1}+y_{2}+\left(y_{1} y_{2}\right)^{2^{n-1}}\right)=y_{1}^{2} y_{2}+y_{1} y_{2}^{2}+y_{1} y_{2} c_{1}^{2^{n}}=0$, leading to

$$
\left(y_{1} y_{2}\right)^{2^{n-1}} c_{2}^{2^{n}}=0 \quad \text { and } \quad c_{1}^{2}=y_{1}^{2}+y_{1} y_{2}+y_{2}^{2} .
$$

So far, everything worked for either $D_{8}$ or $Q_{8}$. Now that we shall use exterior powers, things will start to differ. We have

$$
c_{1}\left(\lambda^{2} \Delta\right)=c_{1}+c_{2}^{2^{n-1}}+c_{1}^{2^{n-1}} c_{2}^{2^{2 n-2}}
$$

since we may calculate modulo $c_{1}^{2^{n}} c_{2}^{2^{n}}$ by (10), and

$$
c_{1}\left(\gamma_{1} \gamma_{2}\right)=y_{1}+y_{2}+\left(y_{1} y_{2}\right)^{2^{n-1}}
$$

hence

$$
c_{1}= \begin{cases}y_{1}+y_{2}+\left(y_{1} y_{2}\right)^{2^{n-1}}+c_{2}^{2^{n-1}}+c_{1}^{2^{n-1}} c_{2}^{2^{2 n-2}} & \text { for } D_{8} \\ c_{2}^{2^{n-1}}+c_{1}^{2^{n-1}} c_{2}^{2^{2 n-2}} & \text { for } Q_{8}\end{cases}
$$


Together with (10) this gives

$$
y_{1}^{2}+y_{1} y_{2}+y_{2}^{2}=c_{1}^{2}= \begin{cases}c_{2}^{2^{n}}+y_{1}^{2}+y_{2}^{2} & \text { for } D_{8}, \\ c_{2}^{2^{n}} & \text { for } Q_{8},\end{cases}
$$

or

$$
c_{2}^{2^{n}}= \begin{cases}y_{1} y_{2} & \text { for } D_{8}, \\ y_{1}^{2}+y_{1} y_{2}+y_{2}^{2} & \text { for } Q_{8} .\end{cases}
$$

For the dihedral group, equations (8), (10) and (13) furthermore imply

$$
\begin{aligned}
c_{1}^{2^{n-1}} c_{2}^{2^{2 n-2}} & =\left(y_{1}^{2}+y_{1} y_{2}+y_{2}^{2}\right)^{2^{n-2}}\left(y_{1} y_{2}\right)^{2^{n-2}} \\
& =\left(\left(y_{1}^{2}+y_{2}^{2}\right) y_{1} y_{2}\right)^{2^{n-2}}+\left(y_{1} y_{2}\right)^{2^{n-1}}=\left(y_{1} y_{2}\right)^{2^{n-1}}
\end{aligned}
$$

whence (11) reads as

$$
c_{1}= \begin{cases}y_{1}+y_{2}+c_{2}^{2^{n-1}} & \text { for } D_{8}, \\ c_{2}^{2^{n-1}}+c_{2}^{2^{2 n-1}} & \text { for } Q_{8} .\end{cases}
$$

Finally, plugging all this into (3) results in

$$
\sum_{k=1}^{n} y_{i}^{2^{n}-2^{k}+1} c_{2}^{2^{k-1}}= \begin{cases}y_{1} y_{2} & \text { for } D_{8}, \\ y_{i}^{2} & \text { for } Q_{8} .\end{cases}
$$

Summing up, we get the following relations:

(1) $y_{i}^{2^{n}}$;

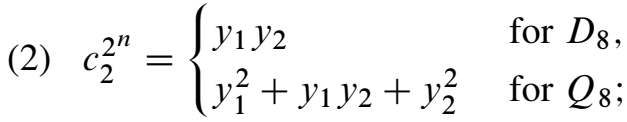

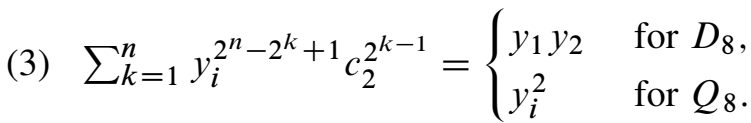

Furthermore, in (14) we have also identified $c_{1}$. Note these relations imply all the others proved along the way, as well as $c_{2}^{2^{2 n-1}+2^{n-1}}=0$.

It remains to check that these relations produce a module of the correct rank, which according to the Euler characteristic formula of Hopkins, Kuhn and Ravenel [4, Theorem B (Part 2)],

$$
\chi_{n, p}(G)=\sum_{A<G} \frac{\mu(A)}{[G: A]} \chi_{n, p}(A)
$$


where summation is over the abelian subgroups of $G$ and $\mu$ is a Möbius function on the poset of abelian subgroups, should be $\frac{3}{2} 4^{n}-\frac{1}{2} 2^{n}$. From the relations one easily reads off that the set (which works for either group)

$$
\mathcal{B}:=\left\{y_{1}^{i} c_{2}^{k}, y_{2}^{j} c_{2}^{l}, c_{2}^{m} \mid 1 \leq i, j<2^{n}, 0 \leq k, l<2^{n-1}, 0 \leq m<\frac{1}{2} 4^{n}+\frac{1}{2} 2^{n}\right\}
$$

generates $C(G ; K(n))$ : by relation $(2)$, we can eliminate any monomial divisible by $y_{1} y_{2}$, and (3) says that $y_{i}^{j+1} c_{2}^{2^{n-1}+k}$ is in the span of $\mathcal{B}$, for any $j, k \geq 0$.

The cardinality of this set, which gives an upper bound for the rank of the Chern approximation, is indeed equal to the rank of $K(n)^{*}(B G)$. Since this is all that is required, we have:

Theorem 4.1 Let $G$ be either $D_{8}$ or $Q_{8}$. Then

(1) $K(n)^{*}(B G) \cong C(G ; K(n))$;

(2) $K(n)^{*}(B G)$ is multiplicatively generated by the classes $y_{1}, y_{2}, c_{2}$ subject to the relations (1)-(3) above.

Remark Note that our relations coincide with those obtained by Bakuradze and Vershinin in [3]. They use slightly different generators though, their $x$ corresponds to our $y_{1}$ and $c$ to $y_{1}+F y_{2}$.

\section{Dihedral, quaternion, and semidihedral groups}

In order to prove a statement like (1) of Theorem 4.1, it is certainly not necessary to determine the complete multiplicative structure.

Suppose one already knew that $K(n)^{*}(B G)$ was generated by Chern classes of representations. It then suffices to produce, using only formal consequences of the ring structure of $R G$ plus Adams and/or exterior power operations, enough relations among the Chern classes of all irreducible representations so that the rank of the result is equal to the Euler characteristic of $G$. This is the course we shall follow from now on; for the assumption on generation by Chern classes we refer to our earlier work [7] and Tezuka and Yagita [11],

Let

$$
G=\left\langle s, t \mid s^{2^{m+1}}=1, t^{2}=s^{e}, t s t^{-1}=s^{r}\right\rangle
$$

where $e \in\left\{0,2^{m}\right\}$ and $r \in\left\{-1,2^{m}-1\right\}$. Then $G \cong D_{2^{m+2}}$, the dihedral group of order $2^{m+2}$, for $e=0$ and $r=-1$, whereas $e=2^{m}, r=-1$ corresponds to the 
generalised quaternion group $Q_{2^{m+2}}$ and $e=0, r=2^{m}-1$ to the semidihedral group $S D_{2^{m+2}}$. Except for this last case, $m=1$ is allowed.

All three types have the same $K(n)$ Euler characteristic

$$
\chi_{n, 2}(G)=\frac{1}{2} 2^{(m+1) n}+4^{n}-\frac{1}{2} 2^{n}
$$

this again follows with the Euler characteristic formula of [4], keeping in mind the following easily verified facts: (i) $G$ has an index two cyclic subgroup, (ii) every other maximal abelian subgroup has order four, (iii) there are $2^{m}+3$ conjugacy classes of elements, and (iv) $\chi_{0, p}(G)=1$ and $\chi_{1, p}(G)$ is the number of conjugacy classes of $p$-elements of $G$, for any group $G$ and prime $p$. Indeed, from (i) and (ii) one has

$$
\chi_{n, 2}(G)=\frac{1}{2} 2^{(m+1) n}+\alpha 4^{n}+\beta 2^{n}
$$

for some $\alpha, \beta$, and solving the equations $\chi_{0,2}(G)=1$ and $\chi_{1,2}(G)=2^{m}+3$ gives the claimed formula.

Furthermore, it is shown eg in [7] that $K(n) *(B G)$ is concentrated in even degrees.

Let $A=\langle s\rangle \cong C_{2^{m+1}}$ and $\rho: A \rightarrow \mathbb{C}^{\times}$be a generator of $R A$ with $\rho(s)=\exp \left(\pi i / 2^{m}\right)$. Define linear characters $\eta_{1}$ and $\eta_{2}$ by

$$
\eta_{1}(s)=-1, \eta_{1}(t)=1 \quad \text { and } \quad \eta_{2}(s)=-1, \eta_{2}(t)=-1 .
$$

The seemingly asymmetric definition will allow us to use the results of the previous section to start inductive arguments. Then $1, \eta_{1}, \eta_{2}$, and $\eta_{1} \eta_{2}$ are the linear characters of $G$. Furthermore, set

$$
\sigma_{k}=\operatorname{Ind}_{A}^{G}\left(\rho^{k}\right) \quad(k \in \mathbb{Z}) .
$$

Note $\sigma_{0}=1+\eta_{1} \eta_{2}$ and $\sigma_{2^{m}}=\eta_{1}+\eta_{2}$ for any of the three types, and $\sigma_{2^{m}+r}=\sigma_{2^{m}-r}$ for $G$ dihedral or quaternion, or $G$ semidihedral and $r$ even, but $\sigma_{2 k+1}=\sigma_{2^{m}-(2 k+1)}$ for $G$ semidihedral and $0 \leq k<2^{m-2}$.

The irreducible two-dimensional complex representations of $G$ are

$$
\begin{aligned}
\sigma_{i}\left(1 \leq i<2^{m}\right) & \text { for } G=D_{2^{m+2}} \text { or } Q_{2^{m+2}} \\
\sigma_{2 j}\left(1 \leq j<2^{m-1}\right), \sigma_{ \pm(2 k+1)}\left(0 \leq k<2^{m-2}\right) & \text { for } G=S D_{2^{m+2}}
\end{aligned}
$$

The next two lemmas give the product structure and Adams and exterior power operations; verification is a routine exercise using complex characters. 
Lemma 5.1 (1) Let $G$ be either dihedral or quaternion. Then $\eta_{i} \sigma_{k}=\sigma_{2^{m}-k}$ $\left(0 \leq k \leq 2^{m}, i=1,2\right)$, and $\sigma_{j} \sigma_{k}=\sigma_{k+j}+\sigma_{k-j}$ for $j \leq k$.

(2) Let $G$ be semidihedral, $i=1,2$, and $0 \leq j \leq k$. Then

$$
\begin{aligned}
\eta_{i} \sigma_{k} & = \begin{cases}\sigma_{2^{m}-k} & k \text { even, } 0 \leq k \leq 2^{m}, \\
\sigma_{-k} & k \text { odd },|k|<2^{m-1},\end{cases} \\
\sigma_{j} \sigma_{k} & = \begin{cases}\sigma_{k+j}+\sigma_{k-j} & j \text { or } k \text { even, } \\
\sigma_{k+j}+\sigma_{2^{m}-k+j} & j \text { and } k \text { odd } .\end{cases}
\end{aligned}
$$

Lemma $5.2 \psi^{k} \sigma_{1}=\sigma_{k}$ for $k$ odd and all three types. Furthermore,

$$
\begin{aligned}
& \psi^{2} \sigma_{k}= \begin{cases}1-\eta_{1} \eta_{2}+\sigma_{2 k} & \text { for } G \cong D_{2^{m+2}}, \text { or } G \cong S D_{2^{m+2}} \text { and } k \text { even, } \\
(-1)^{k}\left(1-\eta_{1} \eta_{2}\right)+\sigma_{2 k} & \text { for } G \cong Q_{2^{m+2}}, \\
\eta_{1}-\eta_{2}+\sigma_{2|k|} & \text { for } G \cong S D_{2^{m+2}} \text { and } k \text { odd },\end{cases} \\
& \lambda^{2} \sigma_{k}= \begin{cases}\eta_{1} \eta_{2} & \text { for } G \cong D_{2^{m+2}} \text { or } k \text { even, } \\
\eta_{2} & \text { for } G \cong S D_{2^{m+2}} \text { and } k \text { odd }, \\
1 & \text { for } G \cong Q_{2^{m+2}} \text { and } k \text { odd } .\end{cases}
\end{aligned}
$$

Now Adams operations can be recovered from exterior powers, thus setting $\sigma=\sigma_{1}$, one has:

Corollary 5.3 (1) $R D_{2^{m+2}}$ and $R S D_{2^{m+2}}$ are generated by $\eta_{1}$ and $\sigma$ as $\Lambda$-rings. (2) $R Q_{2^{m+2}}$ is generated by $\eta_{1}, \eta_{2}$ and $\sigma$ as $\Lambda$-ring.

Theorem 5.4 Let $G$ be either $D_{2^{m+2}}, Q_{2^{m+2}}$, or $S D_{2^{m+2}}$, with $m \geq 2$. Then $C(G, K(n)) \cong K(n)^{*}(B G)$.

Proof Let $y_{i}=c_{1}\left(\eta_{i}\right)$ and $c_{k}=c_{k}(\sigma), k=1,2$. It is known eg from our earlier paper [7] that $K(n)^{*}(B G)$ is generated by $y_{1}, y_{2}$ and $c_{2}$, so all we have to show is that $C(G, K(n))$ has the correct rank.

Since $G /\left\langle s^{4}\right\rangle \cong D_{8}$, we may assume the following relations obtained in Section 4:

$$
y_{1}^{2^{n}}=0, \quad y_{2}^{2^{n}}=0, \quad y_{1}^{2} y_{2}=y_{1} y_{2}^{2} .
$$

Then Lemma 5.2 implies $\psi^{2^{m}} \sigma=1-\eta_{1} \eta_{2}+\eta_{1}+\eta_{2}$; applying $c_{1}$ and $c_{2}$ to this identity yields, using (16),

$$
c_{1}^{2^{m n}}=y_{1}+F y_{2}+y_{1}+y_{2}=\left(y_{1} y_{2}\right)^{2^{n-1}}
$$


and consequently

$$
c_{2}^{2^{m n}}=c_{1}^{2^{m n}}\left(y_{1}+F y_{2}\right)+y_{1} y_{2}=y_{1} y_{2}
$$

since $c_{1}^{2^{n m}}\left(y_{1}+F y_{2}\right)=\left(y_{1} y_{2}\right)^{2^{n-1}}\left(y_{1}+y_{2}+\left(y_{1} y_{2}\right)^{2^{n-1}}\right)=0$ by Lemma 3.1(i) and (16).

The identities for $\lambda^{2} \sigma$ in turn yield, according to Lemma 3.3(v),

$$
c_{1}=Y+c_{2}^{2^{n-1}}+c_{1}^{2^{n-1}} c_{2}^{2^{2 n-2}} \bmod \left(c_{1} c_{2}\right)^{2^{2 n-2}}
$$

with

$$
Y= \begin{cases}y_{1}+F y_{2} & \text { for } G=D_{2^{m+2}} \\ 0 & \text { for } G=Q_{2^{m+2}} \\ y_{2} & \text { for } G=S D_{2^{m+2}}\end{cases}
$$

Next, apply $c_{2}$ to $\eta_{1} \eta_{2} \sigma=\sigma$. Writing $z=y_{1}+F y_{2}=c_{1}\left(\eta_{1} \eta_{2}\right)$, note that by (16) one has $z^{j}=\left(y_{1}+y_{2}\right)^{j}$ for $j>1$. Thus by Lemma 3.2 again,

$$
z c_{1}=z^{2}+S \quad \text { where } S=\sum_{k=1}^{n-1} z^{2^{n}-2^{k}+1} c_{2}^{2^{k-1}}=\sum_{k=1}^{n-1}\left(y_{1}+y_{2}\right)^{2^{n}-2^{k}+1} c_{2}^{2^{k-1}} .
$$

Thus $\left(y_{1}+y_{2}\right) c_{1}=\left(y_{1}+y_{2}\right)^{2}+\left(y_{1} y_{2}\right)^{2^{n-1}} c_{1}+S$. But

$$
\left(y_{1} y_{2}\right)^{2^{n-1}} c_{1}=y_{1}^{2^{n}-1} y_{2} c_{1}=y_{1}^{2^{n}-1}\left(y_{1} c_{1}+\left(y_{1}+y_{2}\right)^{2}+\left(y_{1} y_{2}\right)^{2^{n-1}} c_{1}+S\right)=0 \text {, }
$$

hence

$$
\begin{gathered}
c_{1}^{2^{m n}+1}=0, \\
z c_{1}=\left(y_{1}+y_{2}\right) c_{1}=\left(y_{1}+y_{2}\right)^{2}+S .
\end{gathered}
$$

By Lemma 3.2(v), $z c_{1}^{2}=z^{3}$. With (20) this implies $\left(y_{1}+y_{2}\right) c_{1}^{2^{n}}=z c_{1}^{2^{n}}=z^{2^{n}} c_{1}=0$; in particular, $c_{1}^{2^{2 n-2}} \in \operatorname{ann}\left(y_{1}+y_{2}\right)$ since $n \geq 2$. Thus (18) gives

$$
\left(y_{1}+y_{2}\right) c_{1}=\left(y_{1}+y_{2}\right)\left(Y+c_{2}^{2^{n-1}}+c_{1}^{2^{n-1}} c_{2}^{2^{2 n-2}}\right) .
$$

Together with (20) one obtains

$$
\left(y_{1}+y_{2}\right) c_{2}^{2^{n-1}}=\left(y_{1}+y_{2}\right)^{2}+S+\left(y_{1}+y_{2}\right)\left(Y+c_{1}^{2^{n-1}} c_{2}^{2^{2 n-2}}\right)
$$

or

$c_{2}^{2^{n-1}}=y_{1}+y_{2}+\sum_{k=1}^{n-1}\left(y_{1}+y_{2}\right)^{2^{n}-2^{k}} c_{2}^{2^{k-1}}+Y+c_{1}^{2^{n-1}} c_{2}^{2^{2 n-2}} \bmod \operatorname{ann}\left(y_{1}+y_{2}\right)$ 
whence

$$
c_{2}^{2^{2 n-2}}=\left(y_{1}+y_{2}\right)^{2^{n-1}}+Y^{2^{n-1}}=(z+Y)^{2^{n-1}} \bmod \operatorname{ann}\left(y_{1}+y_{2}\right),
$$

and thus $\left(y_{1}+y_{2}\right) c_{1}^{2^{n-1}} c_{2}^{2^{2 n-2}}=z^{2^{n-1}+1}(z+Y)^{2^{n-1}}=0$ for all three cases. This means we can rewrite (21) as

$$
\left(y_{1}+y_{2}\right) c_{2}^{2^{n-1}}=S+Y^{\prime} \quad \text { with } Y^{\prime}= \begin{cases}0 & \text { for } G=D_{2^{m+2}}, \\ y_{1}^{2}+y_{2}^{2} & \text { for } G=Q_{2^{m+2}}, \\ y_{1}^{2}+y_{1} y_{2} & \text { for } G=S D_{2^{m+2}}\end{cases}
$$

Together with 17.b one then obtains

$$
c_{2}^{2^{(m+1) n-1}+2^{n-1}}=\left(y_{1} y_{2} c_{2}\right)^{2^{n-1}}=y_{1}^{2^{n}-1}\left(y_{1} c_{2}^{2^{n-1}}+S+Y^{\prime}\right)=0 .
$$

Finally, let $\rho=\left(\psi^{2^{m-1}-1} \sigma\right) \cdot\left(\psi^{2^{m-1}} \sigma\right)$. Lemma 5.2 says that $\rho$ is equal to both $\rho^{\prime}=\sigma+\eta_{1} \sigma$ and $\rho^{\prime \prime}=\sigma+\eta_{2} \sigma$. Now $c_{2}(\rho)$ can be expressed as a polynomial in $Y$ and $c_{2}$ : Arguing as before with the splitting principle, we may assume that the two-dimensional representation $\rho$ is a sum $\xi_{1}+\xi_{2}$ of line bundles, whence

$$
\left(\psi^{2^{m-1}-1} \sigma\right) \cdot\left(\psi^{2^{m-1}} \sigma\right)=\left(\xi_{1}^{2^{m-1}-1}+\xi_{2}^{2^{m-1}-1}\right) \cdot\left(\xi_{1}^{2^{m-1}}+\xi_{2}^{2^{m-1}}\right) .
$$

The second Chern class of this expression is a power series in $x_{1}=c_{1}\left(\xi_{1}\right)$ and $x_{2}=c_{1}\left(\xi_{2}\right)$, which is symmetric in $x_{1}$ and $x_{2}$ and thus can be identified with a power series in $c_{1}$ and $c_{2}$. Since all classes are nilpotent, one is left with a polynomial in $c_{1}$ and $c_{2}$. By virtue of (18) and nilpotence again, $c_{1}$ can be written as a polynomial in $Y$ and $c_{2}$, so the same holds for $c_{2}(\rho)$.

On the other hand, by Lemma 3.2 yet again,

$$
\begin{aligned}
c_{2}\left(\sigma+\eta_{j} \sigma\right)=y_{j} c_{1}+y_{j}^{2}+c_{1}^{2}+y_{j}^{2^{n-1}} c_{1}^{2^{n-1}+1} & +y_{j}^{2^{n-1}+1} c_{1}^{2^{n-1}} \\
& +y_{j}^{2^{n-1}} \sum_{k=1}^{n-1} c_{1}^{2^{n-1}-2^{k}+1} c_{2}^{2^{k-1}}
\end{aligned}
$$

for $j=1,2$.

Case $1 G=Q_{2^{m+2}}$. Then $Y=0$, so $c_{2}(\rho)$ is a polynomial $p_{Q}\left(c_{2}\right)$ in $c_{2}$. By (18) and (24),

$$
\begin{array}{ll}
c_{2}\left(\rho^{\prime}\right)=y_{1}^{2}+y_{1} c_{2}^{2^{n-1}} & \bmod \left(c_{2}^{2^{n}}\right), \\
c_{2}\left(\rho^{\prime \prime}\right)=y_{2}^{2}+y_{2} c_{2}^{2^{n-1}} & \bmod \left(c_{2}^{2^{n}}\right),
\end{array}
$$


thus one obtains

$$
\begin{array}{ll}
y_{1} c_{2}^{2^{n-1}}=y_{1}^{2}+p_{Q}\left(c_{2}\right) & \bmod \left(c_{2}^{2^{n}}\right), \\
y_{2} c_{2}^{2^{n-1}}=y_{2}^{2}+p_{Q}\left(c_{2}\right) & \bmod \left(c_{2}^{2^{n}}\right) .
\end{array}
$$

Since all generators are nilpotent, this implies that $y_{1} c_{2}^{2^{n-1}}$ and $y_{2} c_{2}^{2^{n-1}}$ are linear combinations of monomials $y_{i}^{r} c_{2}^{s}$ with $s<2^{n-1}$ whenever $r>0$.

Thus we arrive at the following generating set for $C(Q, K(n))$ :

$$
\left\{y_{i}^{j} c_{2}^{k} \mid i=1,2,1 \leq j<2^{n}, 0 \leq k<2^{n-1}\right\} \cup\left\{c_{2}^{l} \mid 0 \leq l<2^{(m+1) n-1}+2^{n-1}\right\} .
$$

This set has $\chi_{n, 2}(G)$ elements.

Case 2 For $G$ dihedral or semidihedral, it turns out to be more convenient to replace one of the generators $y_{1}, y_{2}$ with $z=y_{1}+{ }_{F} y_{2}$; we shall only treat dihedral groups in detail. So let $G=D_{2^{m+2}}$, then one has $Y=y_{1}+F y_{2}=z$ and

$$
c_{1}=y_{1}+y_{2}+c_{2}^{2^{n-1}} \bmod \left(c_{2}^{2^{2 n-2}}\right)=z+c_{2}^{2^{n-1}} \bmod \left(c_{2}^{2^{2 n-2}}\right) .
$$

Consequently, $c_{2}(\rho)$ is a polynomial $p_{D}\left(z, c_{2}\right)$ in $z$ and $c_{2}$, and we replace the generator $y_{2}$ by $z$. The relevant relations established above then take the form

$$
\begin{aligned}
y_{1}^{2^{n}}=0, \quad z^{2^{n}} & =0, \quad y_{1}^{2} z=y_{1} z^{2}, \\
c_{1}^{2^{m n}} & =\left(y_{1} z\right)^{2^{n-1}}, \\
c_{2}^{2^{m n}} & =y_{1} z+y_{1}^{2},
\end{aligned}
$$

and in particular

$$
z c_{2}^{2^{n-1}}=\sum_{k=1}^{n-1} z^{2^{n}-2^{k}+1} c_{2}^{2^{k-1}}
$$

This means that any polynomial in $z$ and $c_{2}$ can be expressed in terms of monomials $z^{i} c_{2}^{j}$ with $j<2^{n-1}$ whenever $i>0$. 
Now Equation (24) implies, together with $\left(16^{\prime}\right)$ and $\left(17 . b^{\prime}\right)$,

$$
\begin{aligned}
c_{2}\left(\rho^{\prime}\right) & =y_{1}^{2}+c_{1}^{2}+y_{1} c_{1}+\left(y_{1} c_{1}\right)^{2^{n-1}}\left(y_{1}+c_{1}\right)+y_{1}^{2^{n-1}} \sum_{k=1}^{n-1} c_{1}^{2^{n-1}-2^{k}+1} c_{2}^{2^{k-1}} \\
& =y_{1}\left(z+c_{2}^{2^{n-1}}\right)+z^{2}+y_{1}^{2}+\left(y_{1} z+y_{1} c_{2}^{2^{n-1}}\right)\left(y_{1}+y+c_{2}^{2^{n-1}}\right) \\
& +y_{1}^{2^{n-1}} c_{1} \sum_{k=1}^{n-1} z^{2^{n-1}-2^{k}} c_{2}^{2^{k-1}} \bmod \left(c_{2}^{2^{n}}\right) \\
& =z^{2}+y_{1} c_{2}^{2^{n-1}}+\sum_{k=1}^{n-1}\left(y_{1}^{2^{n}-2^{k}+1} c_{2}^{2^{k-1}}+y_{1}^{2^{n}-2^{k}} c_{2}^{2^{k-1}+2^{n-1}} \bmod \left(c_{2}^{2^{n}}\right)\right. \\
& =z^{2}+y_{1} c_{2}^{2^{n-1}}+\sum_{k=1}^{n-1} y_{1}^{2^{n}-2^{k}+1} c_{2}^{2^{k-1}} \bmod \left(c_{2}^{2^{n-1}+1}\right) .
\end{aligned}
$$

Thus equating $c_{2}(\rho)$ and $c_{2}\left(\rho^{\prime}\right)$ yields an equation

$$
y_{1} c_{2}^{2^{n-1}}=\sum_{k=1}^{n-1} y_{1}^{2^{n}-2^{k}+1} c_{2}^{2^{k-1}}+z^{2}+p_{D}\left(z, c_{2}\right) \quad \bmod \left(c_{2}^{2^{n-1}+1}\right)
$$

which as before allows us to express $y_{1} c_{2}^{n-1}$ in terms of monomials $y_{1}^{i} c_{2}^{j}$ and $z^{k} c_{2}^{l}$ with $j<2^{n-1}$ for $i>0$ and $l<2^{n-1}$ for $k>0$, and the set

$$
\left\{y_{1}^{i} c_{2}^{k}, z^{j} c_{2}^{l} \mid 1 \leq i, j<2^{n}, 0 \leq k, l<2^{n-1}\right\} \cup\left\{c_{2}^{r} \mid 0 \leq r<2^{(m+1) n-1}+2^{n-1}\right\}
$$

with $\chi_{n, 2}(G)$ elements generates.

Remark A complete set of relations would be $y_{1}^{2^{n}}=0, y_{2}^{2^{n}}=0, y_{1} y_{2}=c_{2}^{2^{m n}}$,

$$
\left(y_{1}+y_{2}\right) c_{2}^{2^{n-1}}=\sum_{i=1}^{n-1}\left(y_{1}+y_{2}\right)^{2^{n}-2^{i}+1} c_{2}^{2^{i-1}}+ \begin{cases}0 & \text { for } G=D_{2^{m+2}}, \\ y_{1}^{2}+y_{2}^{2} & \text { for } G=Q_{2^{m+2}}, \\ y_{1}^{2}+y_{1} y_{2} & \text { for } G=S D_{2^{m+2}}\end{cases}
$$

and

$$
\begin{aligned}
y_{1} c_{2}^{2^{n-1}}= & \sum_{j=1}^{n-1} y_{1}^{2^{n}-2^{j}+1} c_{2}^{2^{j-1}}+\sum_{k=1}^{m n} c_{2}^{\left(2^{m n}+1\right) 2^{n-1}-\left(2^{n}-1\right) 2^{k-1}} \\
& +\sum_{l=1}^{n-1} c_{2}^{2^{m n-1}\left(2^{n}-2^{l}+1\right)+2^{l-1}}+ \begin{cases}0 & \text { for } G=D_{2^{m+2}}, \\
y_{1}^{2}+y_{1} y_{2} & \text { for } G=Q_{2^{m+2}}, S D_{2^{m+2}} .\end{cases}
\end{aligned}
$$

Such relations were obtained in [3] (in slightly different form). 


\section{Quasidihedral groups}

The quasidihedral group $Q D_{2^{m+2}}$ of order $2^{m+2}$ has a presentation

$$
G=Q D_{2^{m+2}}=\left\langle s, t \mid s^{2^{m+1}}=t^{2}=1, t s t=s^{2^{m}+1}\right\rangle .
$$

Its centre $Z=\left\langle s^{2}\right\rangle$ is cyclic of order $2^{m}$, the commutator subgroup $\left\langle s^{2^{m}}\right\rangle$ has index 2 . The maximal abelian subgroups are $C=\langle s\rangle, C^{\prime}=\langle s t\rangle$, both cyclic of order $2^{m+1}$, and $\left\langle s^{2}, t\right\rangle \cong C_{2^{m}} \times C_{2}$ with common intersection $Z$. Thus the $K(n)$ Euler characteristic is

$$
\chi_{n, 2}(G)=\frac{3}{2} 2^{(m+1) n}-\frac{1}{2} 2^{m n} .
$$

There are $2^{m+1}$ linear characters $\zeta^{r} \eta^{s}, 0 \leq r<2^{m}, s=0,1$, defined by

$$
\zeta(s)=\exp \left(\pi i / 2^{m-1}\right), \zeta(t)=1 \quad \text { and } \quad \eta(s)=1, \eta(t)=-1,
$$

respectively. For reasons to be explained below, we also consider $\xi:=\zeta^{2^{m-1}}$. Furthermore, the group has $2^{m-1}$ irreducible representations of dimension 2: let $\rho \in R C$ be a generator, $\rho(s)=\exp \left(\pi i / 2^{m}\right)$, and set

$$
\sigma_{j}=\operatorname{Ind}_{C}^{G}\left(\rho^{2 j+1}\right), \quad 0 \leq j<2^{m-1} .
$$

This accounts for all irreducible representations. The product structure of $R G$ is

$$
\begin{aligned}
\zeta \sigma_{j} & =\sigma_{j+1}, \quad \eta \sigma_{j}=\sigma_{j}, \\
\sigma_{0}^{2} & =\zeta(1+\xi+\eta+\eta \xi) .
\end{aligned}
$$

Also note that $\xi \sigma_{j}=\sigma_{j}$. Thus $R G$ is generated by $\eta, \zeta$, and $\sigma:=\sigma_{0}$. Finally, one has $\lambda^{2} \sigma=\eta \xi \zeta$. Now set

$$
x=c_{1}(\xi), \quad y=c_{1}(\eta), \quad z=c_{1}(\zeta), \quad c_{1}=c_{1}(\sigma), \quad c_{2}=c_{2}(\sigma) .
$$

(Then $x=z^{2^{(m-1) n}}$. )

From now on let $n \geq 2$. Since $\eta^{2}=\xi^{2}=1$ and $\eta \sigma=\xi \sigma=\sigma$, Lemma 3.2 implies

$$
\begin{gathered}
y^{2^{n}}=0, \quad x^{2^{n}}=0, \\
x c_{1}=x^{2}+\sum_{k=1}^{n-1} x^{2^{n}-2^{k}+1} c_{2}^{2^{k-1}}, \\
y c_{1}=y^{2}+\sum_{k=1}^{n-1} y^{2^{n}-2^{k}+1} c_{2}^{2^{k-1}} .
\end{gathered}
$$


With Lemma 3.3(i), the identity $\sigma^{2}=\zeta(1+\eta+\xi+\eta \xi)$ gives

$$
c_{1}^{2^{n}}=z+\left(y+{ }_{F} z\right)+\left(x+{ }_{F} z\right)+\left(x+{ }_{F} y+{ }_{F} z\right)=(x y)^{2^{n-1}} .
$$

The same identity arises from $\psi^{2} \sigma+\xi \eta \zeta=\zeta+\xi \zeta+\eta \zeta$. As in the previous section, this implies $x(x y)^{2^{n-1}}=y(x y)^{2^{n-1}}=0$ and hence

$$
c_{1}^{2^{n}+1}=0
$$

and $x c_{1}^{2}=x^{3}$ as well as $y c_{1}^{2}=y^{3}$, and finally

$$
x^{2} y=x y^{2} .
$$

Now apply $c_{2}$ and $c_{3}$ to the relation for $\sigma^{2}$; since we may calculate modulo $c_{1}^{2^{n}+1}$, Lemma 3.3 yields

$$
c_{1}^{2}=x^{2}+x y+y^{2}+c_{1}^{2^{n}}\left(z+z^{2^{n}}+c_{2}^{2^{2 n-1}}\right)
$$

and $c_{1}^{2^{n}} c_{2}^{2^{n}}=c_{2}^{2^{n}} z^{2}$.

Next, we use $\lambda^{2} \sigma=\xi \eta \zeta$ and Lemma 3.3(v). Applying $c_{1}$ to this identity shows one can dispense with $z$. Furthermore, since $c_{1}^{2^{n}+1}=0$, one has

(33) $x=c_{1}(\xi)=c_{1}\left(\left(\lambda^{2} \sigma\right)^{2^{m-1}}\right)=\left[2^{m-1}\right] c_{1}\left(\lambda^{2} \sigma\right)= \begin{cases}c_{2}^{2^{m n-1}} & \text { for } m>2, \\ c_{1}^{2^{n}}+c_{2}^{2^{2 n-1}} & \text { for } m=2 .\end{cases}$

Thus the Chern approximation is generated by $y, c_{1}, c_{2}-$ just as $K(n)^{*}\left(B Q D_{2^{m+2}}\right)$, according to [11]. Another generating set would be $\left\{y, z, c_{2}\right\}$. More precisely, from

$$
c_{1}+c_{2}^{2^{n-1}}+c_{2}^{2^{n-1}} c_{2}^{2^{2 n-1}}=c_{1}\left(\lambda^{2} \sigma\right)=c_{1}(\xi \eta \zeta)=x+F y+F_{F} z
$$

one obtains first

$$
\begin{aligned}
z & =c_{1}+x+y+((x+y) z)^{2^{n-1}}+c_{1}^{2^{n}}+c_{2}^{2^{n-1}}+c_{1}^{2^{n-1}} c_{2}^{2^{2 n-2}} \\
& =c_{1}+x+y+(x+y)^{2^{n-1}}\left(c_{1}^{2^{n-1}}+c_{2}^{2^{2 n-2}}\right)+c_{1}^{2^{n}}+c_{2}^{2^{n-1}}+c_{1}^{2^{n-1}} c_{2}^{2^{2 n-2}}
\end{aligned}
$$

and then, using the above expression (32) for $c_{1}^{2}$,

$$
z=c_{1}+x+y+(x+y)^{2^{n-1}} c_{2}^{2^{2 n-2}}+c_{1}^{2^{n}}+c_{2}^{2^{n-1}}+c_{1}^{2^{n-1}} c_{2}^{2^{2 n-2}} .
$$

Plugging this back into (32) finally yields

$$
c_{1}^{2}=x^{2}+x y+y^{2}+c_{1}^{2^{n}} c_{2}^{2^{n-1}}=x^{2}+x y+y^{2}+(x y)^{2^{n-1}} c_{2}^{2^{n-1}} .
$$


The resulting module is thus generated by the set

$$
\begin{aligned}
&\left\{c_{1} c_{2}^{i} \mid 0 \leq i<2^{m n-1}\right\} \cup\left\{c_{2}^{j} \mid 0 \leq j<2^{(m+1) n-1}\right\} \\
& \cup\left\{y^{k} c_{2}^{l} \mid 1 \leq k<2^{n}, 0 \leq l<2^{m n-1}\right\} \cup\left\{y c_{2}^{r} \mid 2^{m n-1} \leq r<2^{(m+1) n-1}\right\}
\end{aligned}
$$

of cardinality $3 \cdot 2^{(m+1) n-1}-2^{m n-1}=\chi_{n, 2}\left(Q D_{2^{m+2}}\right)$. (This set was already shown to be a basis in [1].) We conclude:

Theorem 6.1 The Chern approximation for quasidihedral groups is exact.

Precise relations for $K(n)^{*}\left(B Q D_{2^{m+2}}\right) \cong C\left(Q D_{2^{m+2}} ; K(n)\right)$ are implicit in (27)-(35). They were originally obtained in [1] using transfer methods and $\lambda$-operations.

\section{A 3-primary example}

In this section we present a calculation of $C(G ; K(2))$ for $G$ the nonabelian group of order 27 and exponent 3 , to a significant extent aided by MAPLE. We shall indicate for each individual MAPLE computation which approximation to the formal group law was used and why it suffices.

A presentation of $G$ is

$$
G=\left\langle a, b, c \mid a^{3}=b^{3}=c^{3}=[a, c]=[b, c]=1, b a b^{-1}=a c\right\rangle .
$$

The centre $Z$ of $G$ is $\langle c\rangle \cong C_{3}$ with elementary abelian quotient. There are four maximal abelian subgroups, $\langle a, c\rangle,\langle b, c\rangle,\langle a b, c\rangle,\left\langle a^{2} b, c\right\rangle$, all elementary abelian of rank two and intersecting in the centre. Consequently,

$$
\chi_{n, 3}(G)=\frac{4}{3} 9^{n}-\frac{1}{3} 3^{n} .
$$

There are several ways to calculate its Morava K-theory; either use the split extension $\langle a, c\rangle \rightarrow C \rightarrow C_{3}$ (as done by Kriz; see [5]), or the central extension $\langle c\rangle \rightarrow G \rightarrow C_{p} \times C_{p}$ (as in Tezuka and Yagita; see [10] for $B P$-cohomology). It turns out that $K(n)^{*}(B G)$ is generated by Chern classes (or by transferred Euler classes). Thus this group has the chance of having an exact Chern approximation; we shall however see that this is not so.

First recall the complex representation theory of $G$. Define linear characters $\eta_{1}, \eta_{2}$ by

$$
\eta_{1}(a)=\omega, \quad \eta_{1}(b)=\eta_{1}(c)=1, \quad \eta_{2}(b)=\omega, \quad \eta_{1}(a)=\eta_{2}(c)=1
$$


where $\omega$ is a primitive third root of unity. Furthermore, let $V=\langle a, c\rangle \leq G$, define $\gamma \in R V$ by $\gamma(a)=1, \gamma(c)=\omega$, and set $\sigma_{k}=\operatorname{Ind}_{V}^{G}\left(\gamma^{k}\right)$ for $k=1,2$. The structure of $R G$ as a $\Lambda$-ring is recorded in the following lemma.

Lemma 7.1 (1) The irreducible complex representations of $G$ are $\sigma_{1}, \sigma_{2}$, and $\eta_{1}^{i} \eta_{2}^{j}, 0 \leq i, j \leq 2$.

(2) $\eta_{j}^{3}=1, \eta_{j} \sigma_{k}=\sigma_{k}(j, k=1,2) ; \sigma_{1}^{2}=3 \sigma_{2}, \sigma_{2}^{2}=3 \sigma_{1}, \sigma_{1} \sigma_{2}=\sum_{0 \leq i, j \leq 2} \eta_{1}^{i} \eta_{2}^{j}$.

(3) $\psi^{2} \sigma_{1}=\lambda^{2} \sigma_{1}=\sigma_{2}, \psi^{2} \sigma_{2}=\lambda^{2} \sigma_{2}=\sigma_{1}, \lambda^{3} \sigma_{1}=\lambda^{3} \sigma_{2}=1$.

Thus $\eta_{1}, \eta_{2}$ and $\sigma:=\sigma_{1}$ generate $R G$ as a $\Lambda$-ring. Set $y_{1}=c_{1}\left(\eta_{1}\right), y_{2}=c_{1}\left(\eta_{2}\right)$, and $c_{k}=c_{k}(\sigma)$, then $K(n)^{*}(B G)$ is generated by these classes.

The first relations are easy: $\psi^{3} \eta_{j}=1=\psi^{3} \sigma$ imply

$$
y_{1}^{9}=0, \quad y_{2}^{9}=0, \quad c_{k}^{9}=0 \quad(k=1,2,3) .
$$

Writing (formally) $\sigma=\xi_{1}+\xi_{2}+\xi_{3}$ as a sum of line bundles, and setting $x_{i}=c_{1}\left(\xi_{i}\right)$, we may calculate $c_{1}\left(\lambda^{3} \sigma\right)$ as $c_{1}\left(\xi_{1} \xi_{2} \xi_{3}\right)$. The Witt polynomials $w_{k}$ being polynomials in Chern classes they satisfy $w_{k}^{9}=0$, too. Since $\lambda^{3} \sigma=1$, Ravenel's formula (see Section 3) gives

$$
0=\sum_{n \geq 0}^{F} w_{k}\left(x_{1}, x_{2}, x_{3}\right)^{3^{k}}=w_{0}\left(x_{1}, x_{2}, x_{2}\right)+{ }_{F} w_{1}\left(x_{1}, x_{2}, x_{2}\right)^{3} .
$$

Now $w_{0}=c_{1}$ and $w_{1}=-c_{1} c_{2}+c_{3}$, thus

$$
c_{1}=-c_{3}^{3} .
$$

Next, we evaluate Chern classes of the identities $\eta_{j} \sigma=\sigma, j=1,2$.

Modulo $\left(c_{1}+c_{3}^{3}, c_{2}^{9}, c_{3}^{9}\right)$, one has

$$
\begin{aligned}
& c_{1}\left(\eta_{j} \sigma\right)=c_{1}-y_{j}^{3} c_{2}^{3} \\
& c_{2}\left(\eta_{j} \sigma\right)=c_{2}+y_{j} c_{3}^{3}+y_{j}^{3}\left(c_{2}^{2} c_{3}-c_{2}^{3} c_{3}^{3} c_{3}^{5}\right)+y_{j}^{4} c_{2}^{3}+y_{j}^{6}\left(-c_{2}^{2}+c_{2}^{6}-c_{2} c_{3}^{6}-c_{3}^{4}\right) \\
& \begin{array}{r}
c_{3}\left(\eta_{j} \sigma\right)=c_{3}+y_{j} c_{2}-y_{j}^{2} c_{3}^{3}+y_{j}^{3}\left(1-c_{2} c_{3}^{2}-c_{2}^{2} c_{3}^{4}+c_{3}^{8}\right)+y_{j}^{4}\left(c_{2}^{2} c_{3}-c_{2}^{3} c_{3}^{3}-c_{3}^{5}\right) \\
-y_{j}^{5} c_{2}^{3}+y_{j}^{6}\left(-c_{2} c_{3}-c_{2}^{2} c_{3}^{3}+c_{2}^{5} c_{3}-c_{2}^{3} c_{3}^{5}+c_{3}^{7}\right) \\
+y_{j}^{7}\left(-c_{2}^{2}+c_{2}^{6}-c_{2} c_{3}^{6}-c_{3}^{4}\right) .
\end{array}
\end{aligned}
$$

These expressions were obtained with MAPLE. Since $y_{j}^{9}=0$, it suffices to work with the approximation (i) of Lemma 3.1 for this computation. 
We let MAPLE carry out the calculation in a polynomial ring, use a simple routine to express symmetric polynomials in terms of elementary symmetric functions, ie Chern classes, and reduce modulo relations already obtained. Subsequent computer calculations always follow the same pattern. In particular, when calculating Chern classes of exterior powers or Adams operations, we can express the representations in question as sums of line bundles and calculate in a polynomial algebra.

The equation for $c_{1}$ immediately implies

$$
y_{1}^{3} c_{2}^{3}=y_{2}^{3} c_{2}^{3}=0 .
$$

Using this identity, the equation $c_{2}\left(\eta_{j} \sigma\right)=c_{2}$ simplifies to

$$
0=y_{j} c_{3}^{3}+y_{j}^{3}\left(c_{2}^{2} c_{3}-c_{3}^{5}\right)-y_{j}^{6}\left(c_{2}^{2}+c_{2} c_{3}^{6}+c_{3}^{4}\right),
$$

that is, $c_{3}^{3}=y_{j}^{2}\left(c_{3}^{5}-c_{2}^{2} c_{3}\right)+y_{j}^{5}\left(c_{2}^{2}+c_{2} c_{3}^{6}+c_{3}^{4}\right) \bmod$ ann $\left(y_{j}\right)$. By squaring the latter identity, we obtain $c_{3}^{6}=y_{j}^{4}\left(c_{2}^{4} c_{3}^{2}+c_{2}^{2} c_{3}^{6}\right)=y_{j}^{4} c_{2}^{3} c_{3}^{6}=y_{j}^{6} c_{2}^{4} c_{3}^{6}=0 \bmod \operatorname{ann}\left(y_{j}\right)$ or $y_{j} c_{3}^{6}=0$. Continuing to calculate modulo ann $\left(y_{j}\right)$ one obtains

$$
\begin{aligned}
c_{3}^{5} & =y_{j}^{2}\left(c_{3}^{7}-c_{2}^{2} c_{3}^{3}\right)+y_{j}^{5}\left(c_{2}^{2} c_{3}^{2}+c_{3}^{6}\right)=-y_{j}^{2} c_{2}^{2} c_{3}^{3}+y_{j}^{5} c_{2}^{2} c_{3}^{3} & & \bmod \operatorname{ann}\left(y_{j}\right) \\
& =-y_{j}^{2} c_{2}^{2}\left[y_{j}^{2}\left(c_{3}^{5}-c_{2}^{2} c_{3}\right)+y_{j}^{5}\left(c_{2}^{2}+c_{3}^{4}\right)\right]+y_{j}^{5} c_{2}^{2} c_{3}^{3} & & \bmod \operatorname{ann}\left(y_{j}\right) \\
& =-y_{j}^{4} c_{2}^{2} c_{3}^{5}-y_{j}^{7} c_{2}^{2} c_{3}^{4}+y_{j}^{5} c_{2}^{2} c_{c}^{2} & & \bmod \operatorname{ann}\left(y_{j}\right) \\
& =-y_{j}^{4} c_{2}^{2}\left[-y_{j}^{4} c_{2}^{2} c_{3}^{5}-y_{j}^{7} c_{2}^{2} c_{3}^{4}+y_{j}^{5} c_{2}^{2} c_{3}^{2}\right] & & \\
& \quad-y_{j}^{7} c_{2}^{2} c_{3}\left[y_{j}^{2}\left(c_{3}^{5}-c_{2}^{2} c_{3}\right)+y_{j}^{5}\left(c_{2}^{2}+c_{3}^{4}\right)\right]+y_{j}^{5} c_{2}^{2} c_{3}^{2} & & \bmod \operatorname{ann}\left(y_{j}\right) \\
& =y_{j}^{5} c_{2}^{2} c_{3}^{2} & & \bmod \operatorname{ann}\left(y_{j}\right) .
\end{aligned}
$$

Thus $y_{j} c_{3}^{5}=y_{j}^{6} c_{2}^{2} c_{3}^{2}$ and by a similar calculation, $y_{j}^{6} c_{3}^{4}=-y_{j}^{8} c_{2}^{2} c_{3}^{2}$, hence

$$
y_{j} c_{3}^{3}=-y_{j}^{3} c_{2}^{2} c_{3}+y_{j}^{6} c_{2}^{2} .
$$

These relations furthermore imply $y_{j}^{7} c_{3}^{3}=y_{j}^{4} c_{3}^{5}=0$ and $y_{j} c_{2}^{2} c_{3}^{3}=0$.

The identity for the third Chern class now simplifies to

$$
\begin{aligned}
0 & =y_{j} c_{2}-y_{j}^{2} c_{3}^{3}+y_{j}^{3}\left(1-c_{2} c_{3}^{2}\right)+y_{j}^{4} c_{2}^{2} c_{3}-y_{j}^{6} c_{2} c_{3}+y_{j}^{7} c_{2}^{2} \\
& =y_{j} c_{2}+y_{j}^{4} c_{2}^{3} c_{3}-y_{j}^{7} c_{2}^{2}+y_{j}^{3}\left(1-c_{2} c_{3}^{2}\right)+y_{j}^{4} c_{2}^{2} c_{3}-y_{j}^{6} c_{2} c_{3}+y_{j}^{7} c_{2}^{2} \\
& =y_{j} c_{2}+y_{j}^{3}-y_{j}^{3} c_{2} c_{3}^{2}+y_{j}^{4} c_{2}^{2} c_{3}-y_{j}^{6} c_{2} c_{3} .
\end{aligned}
$$

Applying the resulting equation for $y_{j} c_{2}$ repeatedly then gives

$$
y_{j} c_{2}=-y_{j}^{3}-y_{j}^{5} c_{3}^{2}
$$


which together with (38) implies

$$
y_{j} c_{3}^{3}=-y_{j}^{7} c_{3} .
$$

Rewriting (39) as $c_{2}=-y_{j}^{2}-y_{j}^{4} c_{3}^{3} \bmod$ ann $\left(y_{j}\right)$, taking the square and using (40) yields $y_{j} c_{2}^{2}=y_{j}^{5}-y_{j}^{7} c_{3}^{2}=y_{j}^{5}+y_{j} c_{3}^{4}$, whence

$$
y_{1}^{5} y_{2}-y_{1} y_{2}^{5}=\left(y_{1} c_{2}^{2}+y_{1} c_{3}^{4}\right) y_{2}-y_{1}\left(y_{2} c_{2}^{2}+y_{2} c_{3}^{4}\right)=0
$$

and then

(41) $y_{1}^{3} y_{2}-y_{1} y_{2}^{3}=\left(-y_{1} c_{2}-y_{1}^{5} c_{3}^{2}\right) y_{2}-y_{1}\left(-y_{2} c_{2}-y_{2}^{5} c_{3}^{2}\right)=-\left(y_{1}^{5} y_{2}-y_{1} y_{2}^{5}\right) c_{3}^{2}=0$.

Next, we use $\sigma^{2}=3 \sigma \cdot \lambda^{2} \sigma$ in the form $\psi^{2} \sigma=\lambda^{2} \sigma$. Another MAPLE computation shows that modulo $\left(c_{1}+c_{3}^{3}, c_{2}^{9}, c_{3}^{9}\right)$,

$$
\begin{aligned}
& c_{2}\left(\lambda^{2} \sigma\right)=c_{2}, \quad c_{2}\left(\psi^{2} \sigma\right)=c_{2}+c_{2}^{5}+c_{2}^{3} c_{3}^{4}+c_{2}^{4} c_{3}^{6}-c_{3}^{6}, \\
& c_{3}\left(\lambda^{2} \sigma\right)=-c_{3}, \quad c_{3}\left(\psi^{2} \sigma\right)=-c_{3}+c_{2} c_{3}^{3}-c_{2}^{4} c_{3}+c_{2}^{5} c_{3}^{3}+c_{2}^{6} c_{3}^{5}-c_{2}^{8} c_{3} .
\end{aligned}
$$

For this and the next MAPLE computation it suffices to use an approximation to the formal group law which is accurate up to degree 40 (where the coordinate of the formal group is given degree 1 , so that $c_{i}$ has degree $i$ ); this is good enough since the highest degree nonzero monomial is $c_{2}^{8} c_{3}^{8}$. Such an approximation can be obtained by using Witt polynomials.

The representation ring identity thus gives

$$
\begin{aligned}
c_{2}^{5} & =c_{3}^{6}-c_{2}^{3} c_{3}^{4}-c_{2}^{4} c_{3}^{6}, \\
c_{2} c_{3}^{3} & =c_{2}^{4} c_{3}
\end{aligned}
$$

(for the second equality, observe that $c_{2} c_{3}^{3}=c_{2}^{4} c_{3}-c_{2}^{5} c_{3}^{3}-c_{2}^{6} c_{3}^{5}+c_{2}^{8} c_{3}$ yields $c_{2}^{5} c_{3}^{3}=c_{2}^{8} c_{3}$ and $\left.c_{2}^{6} c_{3}^{3}=0\right)$. Combining (42.a) and (42.b) furthermore yields

$$
c_{2}^{6}=0 \quad \text { and } \quad c_{2}^{5}=c_{3}^{6}
$$

It remains to analyse $\sigma \cdot \lambda^{2} \sigma=\sum_{0 \leq i, j \leq 2} \eta_{1}^{i} \eta_{2}^{j}=: \Sigma$. With the aid of MAPLE once again one obtains $c_{k}\left(\sigma \cdot \lambda^{2} \sigma\right)=0$ for $k=1,3,5,7,8,9$ and

$$
\begin{aligned}
& c_{2}\left(\sigma \cdot \lambda^{2} \sigma\right)=c_{2}^{5}+c_{2}^{3} c_{3}^{4}+c_{2}^{4} c_{3}^{6}+c_{3}^{6}, \\
& c_{4}\left(\sigma \cdot \lambda^{2} \sigma\right)=c_{2}^{5} c_{3}^{6}-c_{2}^{6}\left(1+c_{3}^{8}\right)+c_{2}^{8} c_{3}^{4}, \\
& c_{6}\left(\sigma \cdot \lambda^{2} \sigma\right)=-c_{2}^{2} c_{3}^{6}+c_{2}^{3}\left(1+c_{3}^{8}\right)-c_{2}^{5} c_{3}^{4}+c_{2}^{7}
\end{aligned}
$$


modulo $\left(c_{1}^{3}+c_{3}, c_{2}^{9}, c_{3}^{9}\right)$, which in light of (43) become

$$
c_{k}\left(\sigma \cdot \lambda^{2} \sigma\right)= \begin{cases}-c_{3}^{6} & \text { for } k=2 \\ c_{2}^{3} & \text { for } k=6 \\ 0 & \text { otherwise }\end{cases}
$$

On the other hand, another MAPLE computation (here clearly the approximation of Lemma 3.1(i) to the formal group law suffices) gives $c_{k}(\Sigma)=0$ for $k$ odd and

$$
\begin{aligned}
& c_{2}(\Sigma)=y_{1}^{6} y_{2}^{4}+y_{1}^{4} y_{2}^{6}=-y_{1}^{8} y_{2}^{2}, \\
& c_{4}(\Sigma)=-y_{1}^{6} y_{2}^{6}=0, \\
& c_{6}(\Sigma)=-y_{1}^{6}-y_{2}^{6}-y_{1}^{4} y_{2}^{2}-y_{1}^{2} y_{2}^{4}-y_{1}^{8} y_{2}^{6}-y_{1}^{6} y_{2}^{8}=-y_{1}^{6}-y_{2}^{6}+y_{1}^{4} y_{2}^{2}, \\
& c_{8}(\Sigma)=y_{1}^{6} y_{2}^{2}+y_{1}^{4} y_{2}^{4}+y_{1}^{2} y_{2}^{6}+y_{1}^{8} y_{2}^{8}=0,
\end{aligned}
$$

where we also used (41). Consequently, $c_{3}^{6}=y_{2}^{8} y_{2}^{2}$ and $c_{2}^{3}=-y_{1}^{6}-y_{2}^{6}+y_{1}^{4} y_{2}^{3}$. This exhausts what we can obtain from $R G$. Now define a set $\mathcal{G}:=\left\{f_{1}, f_{2}, \ldots, f_{10}\right\}$ by

$$
\begin{gathered}
f_{1}=y_{1}^{9}, \quad f_{2}=y_{2}^{9}, \quad f_{3}=y_{1} y_{2}^{3}-y_{1}^{3} y_{2}, \quad f_{4}=y_{1} c_{3}^{3}+y_{1}^{7} c_{3}, \\
f_{5}=y_{2} c_{3}^{3}+y_{2}^{7} c_{3}, \quad f_{6}=y_{1} c_{2}+y_{1}^{3}+y_{1}^{5} c_{3}^{2}, \quad f_{7}=y_{2} c_{2}+y_{2}^{3}+y_{2}^{5} c_{3}^{2}, \\
f_{8}=c_{3}^{6}-y_{1}^{8} y_{2}^{2}, \quad f_{9}=c_{2} c_{3}^{3}-\left(y_{1}^{8}+y_{2}^{8}-y_{1}^{6} y_{2}^{2}\right) c_{3}, \quad f_{10}=c_{2}^{3}+y_{1}^{6}+y_{2}^{6}-y_{1}^{4} y_{2}^{2} .
\end{gathered}
$$

Then $\mathcal{G}$ generates the relations ideal. With respect to lexicographic ordering and $c_{2}>c_{3}>y_{2}>y_{1}$, the set $\mathcal{G}$ ist indeed a Gröbner basis; it is not hard to check by hand that all syzygies between the $f_{i}$ reduce to zero modulo $\mathcal{G}$ (or one may trouble MAPLE once more). As a sample calculation we consider the syzygy $s_{9,10}$ between $f_{9}$ and $f_{10}$ : the leading terms are $g_{9}=c_{2} c_{3}^{3}$ and $g_{10}=c_{2}^{3}$, respectively, thus

$$
\begin{aligned}
s_{9,10} & =c_{2}^{2}\left(c_{2} c_{3}^{3}-y_{1}^{8} c_{3}-y_{2}^{8} c_{3}+y_{1}^{6} y_{2}^{2} c_{3}\right)-c_{3}^{3}\left(c_{2}^{3}+y_{1}^{6}+y_{2}^{6}-y_{1}^{4} y_{2}^{2}\right) \\
& =-y_{1}^{8} c_{2}^{2} c_{3}-y_{2}^{8} c_{2}^{2} c_{3}+y_{1}^{6} y_{2}^{2} c_{2}^{2} c_{3}-y_{1}^{6} c_{3}^{3}-y_{2}^{6} c_{3}^{3}+y_{1}^{4} y_{2}^{2} c_{3}^{3}
\end{aligned}
$$

whose summands are divisible by the leading monomials $g_{6}=y_{1} c_{2}, g_{7}=y_{2} c_{2}$, $g_{4}=y_{1} c_{3}^{3}$ and $g_{5}=y_{2} c_{3}^{3}$, whence

$$
\begin{aligned}
s_{9,10} \equiv y_{1}^{10} c_{2} c_{3}+y_{1}^{12} c_{2} c_{3}^{3}+y_{2}^{10} c_{2} c_{3}+y_{2}^{12} c_{2} c_{3}^{3}-y_{1}^{8} y_{2}^{2} c_{2} c_{3} \\
-y_{1}^{10} y_{2}^{2} c_{2} c_{3}^{3}+y_{1}^{12} c_{3}+y_{2}^{12} c_{3}-y_{1}^{10} y_{2}^{2} c_{3} \\
\equiv-y_{1}^{8} y_{2}^{2} c_{2} c_{3} \equiv-y_{1}^{7} y_{2}^{2} c_{3}\left(-y_{1}^{3}-y_{1}^{5} c_{3}^{2}\right) \equiv 0 .
\end{aligned}
$$

The other cases are similar (and shorter). 
Thus one has an additive isomorphism

$$
\begin{aligned}
C(G ; K(2)) \otimes_{K(2) *} \mathbb{F}_{3} \cong \mathbb{F}_{3}\left[y_{1}, y_{2}\right] /\left(y_{1}^{9}, y_{2}^{9}, y_{1} y_{2}^{3}-y_{1}^{3} y_{2}\right) \otimes \mathbb{F}_{3}\left[c_{3}\right] /\left(c_{3}^{3}\right) \\
\oplus \mathbb{F}_{3}\left\{c_{3}^{3}, c_{3}^{4}, c_{3}^{5}, c_{2}, c_{2} c_{3}, c_{2} c_{3}^{2}, c_{2}^{2}, c_{2}^{2} c_{3}, c_{2}^{2} c_{3}^{2}\right\} .
\end{aligned}
$$

Proposition 7.2 Let $G$ be the nonabelian group of order 27 and exponent 3. Then $C(G ; K(2))$ has rank 108 .

Remark A similar result was communicated to the author by Strickland. In the same article where Chern approximations are introduced, he also develops an associated "generalised character theory." In the case at hand, the vector space of "generalised characters" also has rank 108.

To determine the ring structure of $K(2)^{*}(B G)$ for this $G$, one may proceed by the following observations: Firstly, $K(2)^{*}(B G)$ is clearly a quotient of its Chern approximation. Secondly, the spectral sequence calculations alluded to above also give the distribution of additive generators: $K(n)^{*}(B G)$ is "equidistributed" in the sense that $\operatorname{rank}_{K(n)^{*}} K(n)^{2 i}(B G)=\operatorname{rank}_{K(n) *} K(n)^{0}(B G)-1$ for all $i \not \equiv 0 \bmod 2\left(p^{n}-1\right)$. Finally, one may calculate the restrictions to the maximal subgroups. Taken together with the equalities $y_{j} c_{2}^{2}=y_{j}^{5}-y_{j}^{6} c_{3}^{2}$ deduced from $f_{6}$ and $f_{7}$ one obtains

$$
\tilde{f}_{10}:=c_{2}^{2}-\left(y_{1}^{4}+y_{2}^{4}-y_{1}^{2} y_{2}^{2}\right)+\left(y_{1}^{6}+y_{2}^{6}-y_{1}^{4} y_{2}^{2}\right) c_{3}^{2}=0 .
$$

Proposition 7.3 $K(2)^{*}(B G) \cong K(2)^{*}\left[y_{1}, y_{2}, c_{2}, c_{3}\right] /\left(f_{1}, \ldots, f_{9}, \tilde{f}_{10}\right)$.

\section{References}

[1] M Bakuradze, Morava $K$-theory ring for a quasi-dihedral group in Chern classes, $\mathrm{Tr}$. Mat. Inst. Steklova 252 (2006) 31-36 MR2255966

[2] M Bakuradze, S Priddy, Transfer and complex oriented cohomology rings, Alg. Geom. Topol. 3 (2003) 473-509 MR1997326

[3] M Bakuradze, V Vershinin, Morava $K$-theory rings for the dihedral, semidihedral and generalized quaternion groups in Chern classes, Proc. Amer. Math. Soc. 134 (2006) 3707-3714 MR2240687

[4] M J Hopkins, N J Kuhn, D C Ravenel, Generalized group characters and complex oriented cohomology theories, J. Amer. Math. Soc. 13 (2000) 553-594 MR1758754

[5] I Kriz, Morava K-theory of classifying spaces: some calculations, Topology 36 (1997) 1247-1273 MR1452850 
[6] D C Ravenel, Complex cobordism and stable homotopy groups of spheres, Pure and Applied Mathematics 121, Academic Press, Orlando, FL (1986) MR860042

[7] B Schuster, On the Morava K-theory of some finite 2-groups, Math. Proc. Cambridge Philos. Soc. 121 (1997) 7-13 MR1418356

[8] B Schuster, N Yagita, Morava K-theory of extraspecial 2-groups, Proc. Amer. Math. Soc. 132 (2004) 1229-1239 MR2045443

[9] NP Strickland, Chern approximations for generalised group cohomology, Topology 40 (2001) 1167-1216 MR1867242

[10] M Tezuka, N Yagita, Cohomology of finite groups and Brown-Peterson cohomology, from: "Algebraic topology, (Arcata, CA, 1986)", (G Carlsson, R Cohen, H Miller, D Ravenel, editors), Lecture Notes in Math. 1370, Springer, Berlin (1989) 396-408 MR1000392

[11] M Tezuka, N Yagita, Cohomology of finite groups and Brown-Peterson cohomology II, from: "Homotopy theory and related topics, (Kinosaki, 1988)", (M Mimura, editor), Lecture Notes in Math. 1418, Springer, Berlin (1990) 57-69 MR1048175

Fakultät für Mathematik, Ruhr-Universität Bochum

Universitätsstr. 150, D-44801 Bochum, Germany

bjoern.schuster@rub.de

http://www .ruhr-uni-bochum.de/ffm/Lehrstuehle/Lehrstuhl-XIII/

schuster.html

Received: 18 December $2008 \quad$ Revised: 23 May 2012 